\title{
Wilson loops in the light of spin networks
}

\author{
Thierry Lévy *
}

November 21, 2018

\begin{abstract}
If $G$ is any finite product of orthogonal, unitary and symplectic matrix groups, then Wilson loops generate a dense subalgebra of continuous observables on the configuration space of lattice gauge theory with structure group $G$. If $G$ is orthogonal, unitary or symplectic, then Wilson loops associated to the natural representation of $G$ are enough.

This extends a result of A. Sengupta [7. In particular, our approach includes the case of even orthogonal groups.
\end{abstract}

\section{Introduction}

On a compact Lie group, the Peter-Weyl theorem asserts that the characters of irreducible representations generate a dense subalgebra of continuous functions invariant by adjunction. In lattice gauge theory, configuration spaces are powers of a Lie group on which another power of the same group acts, according to the geometry of a given graph and in a way which extends the adjoint action of the group on itself. Peter-Weyl theorem can be adapted to this situation and the functions that play the role of the characters are called spin networks. Despite the fact that spin networks were introduced about forty years ago in a physical context ${ }^{1}$, their importance in lattice gauge theory has been recognized rather recently [1. In the mean time, another set of functions, easier to define, has been used as the standard set of observables: Wilson loops. However, it is not clear at all a priori that this set is complete, that is, that Wilson loops generate a dense subalgebra of continuous invariant functions on the configuration space. A. Sengupta has proved in [7] that it is true when the group is a product of odd orthogonal, unitary (and symplectic) groups. In this paper, an approach similar to that of Sengupta but with a little more classical invariant theory combined with the use of spin networks allows us to add even orthogonal groups to the list and, hopefully, to clarify the argument.

${ }^{*}$ IRMA - 7, rue René Descartes - F-67084 Strasbourg Cedex

${ }^{1}$ R. Penrose introduced them for the purposes of quantization of the geometry of space. See [8] for a historical account. 
The problem of completeness of Wilson loops can be expressed in three equivalent ways. The first one is described above. The second one is more geometrical and consists in asking whether a connection on a principal bundle is determined up to gauge transformation by the conjugacy classes of its loop holonomies. The third one is more algebraic: is it true that the diagonal conjugacy class of a finite collection of elements of a compact Lie group is determined by the conjugacy classes of all possible products one may form with these elements and their inverses ? The equivalence of these questions is discussed in [7, and we will make an important use of the equivalence between the first and the third point of view.

\section{The configuration space}

Let $G$ be a compact connected Lie group. Let $\Gamma=(E, V)$ be a graph with oriented edges. By this we mean that $V$ is a finite set and $E$ is a set of pairs of elements of $V$. Diagonal pairs are allowed and a pair can occur several times in $E$. If $e=(v, w) \in E$ is an edge, we define the source and target of $e$ respectively by $s(e)=v$ and $t(e)=w$. We make the assumption that no vertex is isolated, that is, $s(E) \cup t(E)=V$.

Define an action of $G^{V}$ on $G^{E}$, as follows. For $\phi=\left(\phi_{v}\right)_{v \in V} \in G^{V}$ and $g=\left(g_{e}\right)_{e \in E} \in G^{E}$, set

$$
\phi \cdot g=\left((\phi \cdot g)_{e}\right)_{e \in E} \quad \text { with } \quad(\phi \cdot g)_{e}=\phi_{t(e)}^{-1} g_{e} \phi_{s(e)} .
$$

The configuration space for lattice gauge theory on $\Gamma$ with structure group $G$ is the topological quotient space $\mathcal{C}_{\Gamma}^{G}=G^{V} \backslash G^{E}$ and it can be thought of as a finite-dimensional approximation of a space of connections modulo gauge transformations.

Example 2.1 Consider the very simple graph $\mathrm{L}_{1}$ with one single vertex $v$ and one single edge $(v, v)$. Then $\mathcal{C}_{\mathrm{L}_{1}}^{G}$ is just the space of conjugacy classes on $G$.

Example 2.2 Choose an integer $r \geq 1$ and consider the graph $\mathrm{L}_{\mathrm{r}}$ with $r$ edges depicted below.
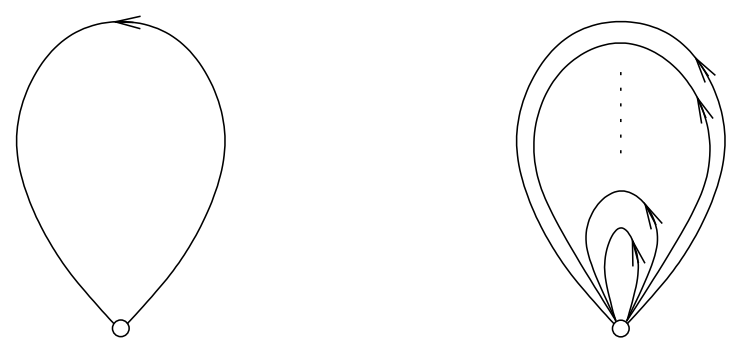

Figure 1: The graphs $L_{1}$ and $L_{r}$.

For this graph, $G^{E}=G^{r}$ on which $G^{V}=G$ acts by diagonal conjugation, and we will call diagonal conjugacy classes of $G^{r}$ the points of $\mathcal{C}_{\mathrm{L}_{\mathrm{r}}}^{G}$. 
Remark 2.3 If $\Gamma$ is a tree, you may check that $\mathcal{C}_{\Gamma}^{G}$ is a single point.

Wilson loops are continuous functions on $\mathcal{C}_{\Gamma}^{G}$ or, equivalently, continuous functions on $G^{E}$ invariant under the action of $G^{V}$. We recall briefly how they are defined.

Let $E^{ \pm}$denote the set containing twice each edge of $\Gamma$, once with its natural orientation and once with the reversed one. Formally, set $E^{ \pm}=E \times\{+,-\}$, extend the functions $s$ and $t$ to $E^{ \pm}$by $s(e,+)=s(e), s(e,-)=t(e)$ and the two similar rules for $t$. A point of $G^{E}$ determines a point of $G^{E^{ \pm}}$by the rules $g_{(e,+)}=g_{e}$ and $g_{(e,-)}=g_{e}^{-1}$. For the sake of clarity, we identify $e$ with $(e,+)$ and denote $(e,-)$ by $e^{-1}$. Moreover, we use the notation $e$ to denote a generic element of $E^{ \pm}$.

A path in $\Gamma$ is a finite sequence $p=\left(e_{1}, \ldots, e_{n}\right)$ of elements of $E^{ \pm}$such that $t\left(e_{i}\right)=$ $s\left(e_{i+1}\right)$ for all $i=1, \ldots, n-1$. It is a loop based at $v$ if $t\left(e_{n}\right)=s\left(e_{1}\right)=v$. To a loop $l=\left(e_{1}, \ldots, e_{n}\right)$ one associates a function $h_{l}: G^{E} \longrightarrow G$ defined by $h_{l}(g)=g_{e_{n}} \ldots g_{e_{1}}$.

One checks easily that the action of $\phi \in G^{V}$ on $G^{E}$ conjugates $h_{l}$ by $\phi_{s\left(e_{1}\right)}^{-1}$ so that, given any finite-dimensional representation $\alpha$ of $G$ with character $\chi_{\alpha}$, the function

$$
W_{\alpha, l}=\chi_{\alpha} \circ h_{l}: \mathcal{C}_{\Gamma}^{G} \longrightarrow \mathbb{C}
$$

is well-defined. It is called a Wilson loop.

Remark 2.4 A wider class of functions can be defined on $\mathcal{C}_{\Gamma}^{G}$. Instead of considering one loop, we can consider several loops $l_{1}, \ldots, l_{n}$ based at the same point. Then, for any function $f: G^{n} \longrightarrow \mathbb{C}$ invariant by diagonal adjunction, that is, such that for all $g_{1}, \ldots, g_{n}, h \in G$, one has $f\left(g_{1}, \ldots, g_{n}\right)=f\left(h g_{1} h^{-1}, \ldots, h g_{n} h^{-1}\right)$, the function

$$
f \circ\left(h_{l_{1}}, \ldots, h_{l_{n}}\right): \mathcal{C}_{\Gamma}^{G} \longrightarrow \mathbb{C}
$$

is well-defined. In words, the diagonal conjugacy class of $\left(h_{l_{1}}(c), \ldots, h_{l_{n}}(c)\right)$ is well-defined for every $c$ in the configuration space.

\section{Statement of the result}

In this paper, $O(n)$ and $S O(n)$ denote respectively the groups $O_{n} \mathbb{R}$ and $S O_{n} \mathbb{R}$. By the symplectic group $S p(n)$ we mean the $\operatorname{subgroup}^{2} U(2 n) \cap S p_{2 n} \mathbb{C}$ of $G L_{2 n} \mathbb{C}$. It is isomorphic to the quaternionic unitary group $U_{\mathbb{H}}(n)$. The main result is the following.

Theorem 3.1 Let $G$ be a finite product of groups among $U(n), S U(n), O(n), S O(n)$, $S p(n)$. Let $\Gamma=(E, V)$ be a graph. Then the algebra generated by the Wilson loops is dense in the space of continuous functions on $\mathcal{C}_{\Gamma}^{G}=G^{E} / G^{V}$.

Example 3.2 In the case of the graph $\mathrm{L}_{1}$, Theorem 3.1 is equivalent to Peter-Weyl theorem.

\footnotetext{
${ }^{2} S p_{2 n} \mathbb{C}$ is the group of matrices which preserve the skew-symmetric form whose matrix in the canonical basis is $\left(\begin{array}{cc}0 & I \\ -I & 0\end{array}\right)$.
} 
Example 3.3 Consider the case of the graph $L_{r}$. Loops in $L_{r}$ are in one-to-one correspondence with words in the letters of $E^{ \pm}=\left\{e_{1}^{ \pm 1}, \ldots, e_{r}^{ \pm 1}\right\}$. For such a word $w$ and given a point $g=\left(g_{1}, \ldots, g_{r}\right)$ of $G^{E}$, let us denote by $w(g)$ the corresponding product in reversed order of the $g_{i}^{\prime} s$ and their inverses. Observe that, if a loop $l$ corresponds to a word $w$, then $h_{l}(g)=w(g)$ for all $g$.

Assume for a moment that Theorem 3.1 is proved for the graphs $\mathrm{L}_{\mathrm{r}}$. We can rephrase it as follows.

Proposition 3.4 Let $G$ be a group as in Theorem [3.1. If $g$ and $g^{\prime}$ are two points of $G^{r}$ such that for all word $w$ in $r$ letters and their inverses, the elements $w(g)$ and $w\left(g^{\prime}\right)$ of $G$ are conjugate, then $g$ and $g^{\prime}$ belong to the same diagonal conjugacy class.

Proof. In this proof, we identify freely $G^{r}$ with $G^{E}$, where $E$ is the set of edges of the graph $\mathrm{L}_{\mathrm{r}}$. If two points $g$ and $g^{\prime}$ of $G^{r}$ do not belong to the same diagonal conjugacy class, their orbits in $\mathcal{C}_{\mathrm{L}_{\mathrm{r}}}^{G}$ are different. Hence, by Theorem 3.1 applied to the graph $\mathrm{L}_{\mathrm{r}}$, there exists a loop $l$ in $\mathrm{L}_{\mathrm{r}}$ such that $h_{l}(g)$ and $h_{l}\left(g^{\prime}\right)$ are not conjugate. This loop is a word $w$ in the letters of $E^{ \pm}$and the corresponding elements $w(g)$ and $w\left(g^{\prime}\right)$, which are precisely $h_{l}(g)$ and $h_{l}\left(g^{\prime}\right)$, are not conjugate.

It turns out that Proposition 3.4 is almost equivalent to Theorem 3.1. The gap is filled by the following result.

Proposition 3.5 Let $G$ be a compact group. Let $\Gamma=(E, V)$ be a graph. Let $c$ and $c^{\prime}$ be two points of $\mathcal{C}_{\Gamma}^{G}$. Assume that, for any vertex $v$ of $V$ and any finite sequence $l_{1}, \ldots, l_{r}$ of loops in $\Gamma$ based at $v$, the diagonal conjugacy classes of $\left(h_{l_{1}}(c), \ldots, h_{l_{r}}(c)\right)$ and $\left(h_{l_{1}}\left(c^{\prime}\right), \ldots, h_{l_{r}}\left(c^{\prime}\right)\right)$ are equal. Then $c=c^{\prime}$.

This proposition is proved in a slightly different language in [6]. For the convenience of the reader, we recall the argument.

Proof. Fix once for all a vertex $v$. Choose $g$ and $g^{\prime}$ in $G^{E}$ representing $c$ and $c^{\prime}$. For any finite family $F$ of loops based at $v$, let $K_{F}$ be the closed subset of $G$ consisting of those $k$ such that $h_{l}\left(g^{\prime}\right)=k h_{l}(g) k^{-1}$ for all $l \in F$. By assumption, $K_{F}$ is non-empty, just as any finite intersection of sets of the form $K_{F}$. By compactness of $G$, there exists $k$ such that $h_{l}\left(g^{\prime}\right)=k h_{l}(g) k^{-1}$ for every loop $l$ based at $v$. By letting the element of $G^{V}$ equal to $k$ at $v$ and 1 anywhere else act on $g^{\prime}$, we are reduced to the case where $h_{l}(g)=h_{l}\left(g^{\prime}\right)$ for all $l$ based at $v$.

Now, for every vertex $w$, choose a path $p$ in $\Gamma$ joining $w$ to $v$. Define $\phi_{w}=h_{p}(g) h_{p^{-1}}\left(g^{\prime}\right)$. Then one checks easily that $\phi_{w}$ does not depend on $p$ and that the element $\phi=\left(\phi_{w}\right)_{w \in V}$ of $G^{V}$ built in that way satisfies $\phi \cdot g=g^{\prime}$. Hence, $c=c^{\prime}$.

We have reduced the problem as follows.

Proposition 3.6 Theorem 3.1 is logically equivalent to its specialization to the graphs $\mathrm{L}_{\mathrm{r}}, \mathrm{r} \geq 1$, which is in turn equivalent to Proposition 3.4. 
Proof. We prove that Proposition 3.4 implies Theorem 3.1. Let $\Gamma$ be a graph. Let $g$ and $g^{\prime}$ be two points of $G^{E}$ such that all Wilson loops take the same value at $g$ and $g^{\prime}$. Let $v$ be a vertex of the graph and $l_{1}, \ldots, l_{r} r$ loops based at $v$. Since any product of the $l_{i}$ 's and their inverses is still a loop based at $v$, Proposition 3.4 applied to the elements $\left(h_{l_{1}}(g), \ldots, h_{l_{r}}(g)\right)$ and $\left(h_{l_{1}}\left(g^{\prime}\right), \ldots, h_{l_{r}}\left(g^{\prime}\right)\right)$ of $G^{r}$ shows that there exists $k \in G$ such that $h_{l_{i}}\left(g^{\prime}\right)=k h_{l_{i}}(g) k^{-1}$ for all $i=1 \ldots r$. Hence, by Proposition 3.5. $g$ and $g^{\prime}$ belong to the same orbit under the action of $G^{V}$. Hence, Wilson loops separate the points on the configuration space. Since this space is compact, the result follows by the Stone-Weierstrass theorem.

The translation in algebraic language allows us to reduce the list of groups that we need to consider. The proof of the following lemma is straightforward.

Lemma 3.7 If Proposition 3.4 holds for two groups $G_{1}$ and $G_{2}$, then it holds for their product $G_{1} \times G_{2}$.

According to this lemma, it is enough to prove Theorem 3.1 when $G$ is one of the groups $O(n), S O(n), U(n), S U(n), S p(n)$.

Remark 3.8 One might expect that the property expressed by Proposition 3.4 is preserved by standard transformations of the group such as quotients or central extensions. Unfortunately, no such result seems easy to prove. For central extensions, A. Sengupta has stated and proved in [7] a partial result, namely that a property slightly stronger than that of Proposition 3.4 is preserved. I have not been able to improve this result.

\section{Spin networks}

From now on, we concentrate on the case where $\Gamma$ is the graph $L_{r}$ for some $r \geq 1$ and $G$ is one of the groups listed above. Instead of working on the configuration space, we prefer to work on $G^{E}=G^{r}$ and consider only objects which are invariant under the diagonal adjoint action of $G$.

Spin networks provide us with a very natural dense subalgebra of the space of invariant continuous functions. They are defined as follows.

Choose $r$ finite-dimensional representations $\alpha_{1}, \ldots, \alpha_{r}$ of $G$ with spaces $V_{1}, \ldots, V_{r}$. Then $G$ acts on $V_{1} \otimes \ldots \otimes V_{r}$ by $\alpha_{1} \otimes \ldots \otimes \alpha_{r}$. Let us choose $I \in \operatorname{End}_{G}\left(V_{1} \otimes \ldots \otimes V_{r}\right)$. This means that $I$ is a linear endomorphism of $V_{1} \otimes \ldots \otimes V_{r}$ commuting with the action of $G$. Let $g$ be an element of $G^{r}$. Set $\boldsymbol{\alpha}=\left(\alpha_{1}, \ldots, \alpha_{r}\right)$. Then the function $\psi_{\boldsymbol{\alpha}, I}: G^{r} \longrightarrow \mathbb{C}$ defined by

$$
\psi_{\boldsymbol{\alpha}, I}(g)=\operatorname{tr}\left(\alpha_{1}\left(g_{1}\right) \otimes \ldots \otimes \alpha_{r}\left(g_{r}\right) \circ I\right)
$$

is invariant under the action of $G$. It is called a spin network.

The following proposition has been proved by J. Baez [1]. 
Theorem 4.1 The spin networks $\psi_{\boldsymbol{\alpha}, I}$, where $\boldsymbol{\alpha}$ runs over the set of $r$-tuples of irreducible representations of $G$ and, given $\boldsymbol{\alpha}=\left(\alpha_{1}, \ldots, \alpha_{r}\right)$, I runs over a basis of $\operatorname{End}_{G}\left(V_{1} \otimes \ldots \otimes\right.$ $\left.V_{r}\right)$, generate a dense subalgebra of $C\left(G^{r}\right)^{G}$, the space of continuous functions invariant under the diagonal action of $G$.

Remark 4.2 Just as in Peter-Weyl theorem, there is also a $L^{2}$ version of this result, but we do not need it here.

For the sake of completeness and because we find it illuminating, we give a short proof of Theorem 4.1

Proof. The irreducible representations of $G^{r}$ are exactly the tensor products of $r$ irreducible representations of $G$. Thus, Peter-Weyl theorem applied to $G^{r}$ implies that the functions $\psi_{\boldsymbol{\alpha}, J}$ on $G^{r}$, where $\boldsymbol{\alpha}$ is as before, but $J$ is any endomorphism of $V_{1} \otimes \ldots \otimes V_{r}$, generate a dense subalgebra of $C\left(G^{r}\right)$.

Now, it is readily seen that the average under the diagonal action of $G$ of such a function $\psi_{\boldsymbol{\alpha}, J}$ is a spin network $\psi_{\boldsymbol{\alpha}, I}$, where $I$ is the orthogonal projection of $J$ on $\operatorname{End}_{G}\left(V_{1} \otimes \ldots \otimes V_{r}\right)$ for any $G$-invariant scalar product on $\operatorname{End}\left(V_{1} \otimes \ldots \otimes V_{r}\right)$. The result follows immediately.

Let us call the spin network $\psi_{\boldsymbol{\alpha}, I}$ irreducible if $\boldsymbol{\alpha}$ is irreducible as a representation of $G^{n}$, that is, if every $\alpha_{i}$ is irreducible.

Proposition 4.3 Any spin network is a linear combination of irreducible spin networks.

Proof. Let $\psi_{\boldsymbol{\alpha}, I}$ be a spin network. Decompose $\boldsymbol{\alpha}$ as a sum $\bigoplus_{k} \boldsymbol{\alpha}_{k}$ of irreducible representations of $G^{n}$. Accordingly, decompose the space $V$ of $\boldsymbol{\alpha}$ as $V=\bigoplus_{k} V_{k}$. For each $k$, define $I_{k}$ as the component of $I$ lying in $\operatorname{End}_{G}\left(V_{k}\right)$ in the decomposition

$$
\operatorname{End}_{G}(V)=\operatorname{End}_{G}\left(\bigoplus_{k} V_{k}\right) \simeq \bigoplus_{k, l} \operatorname{Hom}_{G}\left(V_{k}, V_{l}\right) .
$$

Then we leave it for the reader to check that $\psi_{\boldsymbol{\alpha}, I}=\sum_{k} \psi_{\boldsymbol{\alpha}_{k}, I_{k}}$.

In order to establish Theorem 3.1 for the graphs $\mathrm{L}_{\mathrm{r}}$, it is thus enough to prove the following result.

Proposition 4.4 Let $G$ be one of the groups $O(n), S O(n), U(n), S U(n), S p(n)$. Let $r \geq 1$ be an integer. On the graph $\mathrm{L}_{\mathrm{r}}$, any irreducible spin network is a finite linear combination of products of Wilson loops.

We have now almost reached the formulation of the problem under which we are going to solve it. 


\section{$5 \quad$ Natural representations}

The main problem we are going to encounter in handling with spin networks is that they involve invariant endomorphisms of spaces of representations of $G$, which are in general very difficult to describe.

In the case where $G$ is a group of complex matrices of some size $n$, that is, an orthogonal, unitary or symplectic group ${ }^{3}, G$ acts by left multiplication on $V=\mathbb{C}^{n}$ and this is called the natural representation. The contragredient of this representation is the action on $V^{*}$ given by $g \cdot \varphi=\varphi \circ g^{-1}$.

The first fundamental theorems (FFT) of classical invariant theory describe a set of generators of the space $\operatorname{End}_{G}\left(V^{\otimes p} \otimes\left(V^{*}\right)^{\otimes q}\right)$ when $p$ and $q$ are given integers, for the different kinds of matrix groups $G$.

This gives us what we are looking for in a special case, namely when each representation $\alpha_{i}$ is of the form $V^{\otimes p} \otimes\left(V^{*}\right)^{\otimes q}$. The two following results allow us to reduce the general case to this particular one.

Lemma 5.1 Let $G$ be any compact Lie group. Consider $\boldsymbol{\alpha}=\left(\alpha_{1}, \ldots, \alpha_{r}\right)$ and $\boldsymbol{\beta}=$ $\left(\beta_{1}, \ldots, \beta_{r}\right)$ two $r$-tuples of representations of $G$. Assume that, for each $i=1 \ldots r$, the representation $\alpha_{i}$ is a subrepresentation of $\beta_{i}$. Let $\psi_{\boldsymbol{\alpha}, I}$ be a spin network on $G^{r}$. Then there exists $J \in \operatorname{End}_{G}\left(\beta_{1} \otimes \ldots \otimes \beta_{r}\right)$ such that $\psi_{\boldsymbol{\alpha}, I}=\psi_{\boldsymbol{\beta}, J}$.

Proof. For each $i$, endow the space of $\beta_{i}$ with a $G$-invariant scalar product and define $p_{i}$ as the orthogonal projection on a subspace on which the action of $G$ is isomorphic to $\alpha_{i}$. Then $J=I \circ p_{1} \otimes \ldots \otimes p_{r}$ is $G$-invariant and satisfies $\psi_{\boldsymbol{\alpha}, I}=\psi_{\boldsymbol{\beta}, J}$.

Proposition 5.2 Let $G$ be a compact Lie group. Let $\alpha$ be a faithful finite-dimensional representation of $G$. Then any irreducible representation of $G$ is a subrepresentation of $\alpha^{\otimes p} \otimes\left(\alpha^{\vee}\right)^{\otimes q}$ for some integers $p, q \geq 0$.

In this statement, $\alpha^{\vee}$ denotes the contragredient representation of $\alpha$. We use the convention $\alpha^{\otimes 0}=\mathbb{C}$, the trivial representation.

This result is of course well-known ${ }^{4}$ in the sense that the representations of compact Lie groups are completely classified and that a proof "by inspection" is almost possible, see for example the end of [2]. However, we were not able to find a direct proof in textbooks on Lie groups. Therefore, we propose a short analytical argument.

Proof. Let $\alpha$ be a faithful finite-dimensional representation of $G$. Since $\alpha$ is unitary for some Hermitian scalar product, its character satisfies the inequality $\left|\chi_{\alpha}(g)\right| \leq \chi_{\alpha}(1)$ with equality only if $\alpha(g)= \pm \mathrm{Id}$. Hence, $\left|\chi_{\alpha}(g)+1\right|$ is maximal only when $\alpha(g)=\mathrm{Id}$, that

\footnotetext{
${ }^{3}$ Recall that the elements of $S p(n)$ are complex matrices of size $2 n$.

${ }^{4}$ In the case of a finite group, it is referred to as a theorem of Burnside and Molien in 3 .
} 
is, since $\alpha$ is faithful, when $g=e$, the identity element of $G$. This implies immediately that the probability measures

$$
\mu_{n}=\frac{\left|\chi_{\alpha}(g)+1\right|^{2 n}}{\int_{G}\left|\chi_{\alpha}(g)+1\right|^{2 n} d g} d g
$$

on $G$ converge weakly to the Dirac mass $\delta_{e}$. Here, $d g$ denotes the unit-mass Haar measure on $G$. In particular, let $\rho$ be any irreducible representation of $G$. Since $\mu_{n}\left(\chi_{\rho}\right)$ converges to $\chi_{\rho}(e) \neq 0$, there exists an integer $n \geq 0$ such that

$$
\int_{G} \chi_{\rho}(g)\left|\chi_{\alpha}(g)+1\right|^{2 n} d g \neq 0
$$

Now observe that $\left|\chi_{\alpha}+1\right|^{2}$ is just the character of the representation $(\alpha \oplus \mathbb{C}) \otimes(\alpha \oplus \mathbb{C})^{\vee} \simeq$ $\mathbb{C} \oplus \alpha \oplus \alpha^{\vee} \oplus\left(\alpha \otimes \alpha^{\vee}\right)$, where $\mathbb{C}$ denotes the trivial representation of $G$. Thus, $\rho$ is a subrepresentation of the $n$-th tensor product of this representation. This tensor product breaks into (non-necessarily irreducible) factors of the form $\alpha^{\otimes p} \otimes\left(\alpha^{\vee}\right)^{\otimes q}$, so that $\rho$, being irreducible, is a subrepresentation of one of them.

Remark 5.3 We have not used the fact that $G$ was a Lie group, we have only used its compactness. However, a compact group admits a faithful finite-dimensional representation if and only if it is a Lie group (see for example [5]).

For matrix groups, Proposition 5.2 ensures that every irreducible representation arises as a subrepresentation of some tensor product of a number of copies of the natural representation and its contragredient. We are now reduced to prove the following result.

Proposition 5.4 Let $G$ be a group of the following list: $O(n), S O(n), U(n), S U(n)$, $S p(n)$. Let $r \geq 1$ be an integer. Let $\boldsymbol{\alpha}$ be a $r$-tuple of representations of the form $V^{\otimes p} \otimes\left(V^{*}\right)^{\otimes q}$, where $V$ is the natural representation of $G$. Then any spin network $\psi_{\boldsymbol{\alpha}, I}$ on $G^{r}$ is a linear combination of products of Wilson loops.

We leave it to the reader to check that Proposition 5.4 implies Propostion 4.4 .

\section{$6 \quad$ Unitary groups}

Let $n \geq 1$ be an integer and let $G$ be either $U(n)$ or $S U(n)$. The group $G$ acts on $V=\mathbb{C}^{n}$ by multiplication on the left. For any integer $d \geq 1$, there is a corresponding diagonal action of $G$ on $V^{\otimes d}$, that we denote by $\rho: G \longrightarrow G L\left(V^{\otimes d}\right)$. On the other hand, the symmetric group $\mathfrak{S}_{d}$ acts by permutation of the factors on $V^{\otimes d}$. We denote this action by $\pi: \mathfrak{S}_{d} \longrightarrow G L\left(V^{\otimes d}\right)$. It is obvious that the actions $\rho$ and $\pi$ commute to each other. The following theorem is known as Schur-Weyl duality theorem. 
Theorem 6.1 (Schur-Weyl duality) The two subalgebras $\rho(\mathbb{C} U(n))$ and $\pi\left(\mathbb{C S}_{d}\right)$ of $\operatorname{End}\left(V^{\otimes d}\right)$ are each other's commutant.

In other words, $\operatorname{End}_{U(n)}\left(V^{\otimes d}\right)$ is generated as a vector space by the permutations of the factors. The case of $S U(n)$ follows immediately, since $\operatorname{End}_{S U(n)}\left(V^{\otimes d}\right)=\operatorname{End}_{U(n)}\left(V^{\otimes d}\right)$.

Proof. By the bicommutant theorem (see [4] for example), it is equivalent to prove that $\pi\left(\mathbb{C S}_{d}\right)^{\prime}=\rho(\mathbb{C} G)$ or to prove that $\rho(\mathbb{C} G)^{\prime}=\pi\left(\mathbb{C S}_{d}\right)$. The second statement is the most important for us, but the first one is the easiest to prove.

By definition, $\pi\left(\mathbb{C S}_{d}\right)^{\prime}=\operatorname{End}_{\mathfrak{S}_{d}}\left(V^{\otimes d}\right)$, which in turn is just $\operatorname{End}\left(V^{\otimes d}\right)^{\mathfrak{S}_{d}}$, where $\mathfrak{S}_{d}$ acts by conjugation on $\operatorname{End}\left(V^{\otimes d}\right)$. Now,

$$
\operatorname{End}\left(V^{\otimes d}\right)^{\mathfrak{S}_{d}} \simeq\left[\operatorname{End}(V)^{\otimes d}\right]^{\mathfrak{S}_{d}} \simeq \operatorname{Sym}^{d}(\operatorname{End}(V)) .
$$

We must prove that $\operatorname{Sym}^{d}(\operatorname{End}(V))$ is generated by the endomorphisms of the form $\rho(g)^{\otimes d}$, $g \in U(n)$. This is true because $U(n)$ is Zariski-dense in $\operatorname{End}\left(\mathbb{C}^{n}\right)$ and, for any finitedimensional vector space $W$, $\operatorname{Sym}^{d}(W)$ is generated by $\left\{x^{\otimes d} \mid x \in X\right\}$ as soon as $X$ is Zariski-dense $^{5}$ in $W$.

Consider the following isomorphisms of $G$-modules:

$$
\begin{aligned}
\operatorname{End}\left(V^{\otimes p} \otimes\left(V^{*}\right)^{\otimes q}\right) & \simeq\left(V^{*}\right)^{\otimes p} \otimes V^{\otimes p} \otimes V^{\otimes q} \otimes\left(V^{*}\right)^{\otimes q} \\
& \simeq\left(V^{*}\right)^{\otimes p+q} \otimes V^{\otimes p+q} \\
& \simeq \operatorname{End}\left(V^{\otimes p+q}\right),
\end{aligned}
$$

where the second one is chosen in the simplest possible way, namely

$$
\varphi_{1} \ldots \varphi_{p} u_{1} \ldots u_{p} v_{1} \ldots v_{q} \psi_{1} \ldots \psi_{q} \mapsto \varphi_{1} \ldots \varphi_{p} \psi_{1} \ldots \psi_{q} u_{1} \ldots u_{p} v_{1} \ldots v_{q} .
$$

If $\sigma$ belongs to $\mathfrak{S}_{p+q}$, let us denote by $I_{\sigma}$ the element of $\operatorname{End}\left(V^{\otimes p} \otimes\left(V^{*}\right)^{\otimes q}\right)$ corresponding via (III) to $\pi(\sigma)$. Schur-Weyl duality implies that $\operatorname{End}_{G}\left(V^{\otimes p} \otimes\left(V^{*}\right)^{\otimes q}\right)$ is generated by the endomorphisms $I_{\sigma}$.

Let $p_{1}, \ldots, p_{r}, q_{1}, \ldots, q_{r}$ be non-negative integers. For each $i=1 \ldots r$, consider the representation $\alpha_{i}=V^{\otimes p_{i}} \otimes\left(V^{*}\right)^{\otimes q_{i}}$ of $G$ and set $\boldsymbol{\alpha}=\left(\alpha_{1}, \ldots, \alpha_{r}\right)$. Set $p=p_{1}+\ldots+p_{r}$ and $q=q_{1}+\ldots+q_{r}$. Let $\sigma$ be an element of $\mathfrak{S}_{p+q}$. Consider $I_{\sigma} \in \operatorname{End}_{G}\left(V^{\otimes p} \otimes\left(V^{*}\right)^{\otimes q}\right)$. By the isomorphism $V^{\otimes p} \otimes\left(V^{*}\right)^{\otimes q} \simeq \otimes_{i=1}^{r}\left(V^{\otimes p_{i}} \otimes\left(V^{*}\right)^{\otimes q_{i}}\right), I_{\sigma}$ can be seen as an element of $\operatorname{End}_{G}\left(\alpha_{1} \otimes \ldots \otimes \alpha_{r}\right)$. We may thus form the spin network $\psi_{\boldsymbol{\alpha}, I_{\sigma}}$. The following proposition implies Proposition 5.4 in the case of unitary groups.

Proposition 6.2 The spin network $\psi_{\boldsymbol{\alpha}, I_{\sigma}}$ on $G^{r}$ is a product of Wilson loops.

\footnotetext{
${ }^{5}$ This is most easily seen through the identification $\operatorname{Sym}^{d}(W) \simeq \mathcal{P}^{d}(W)^{*}$, where $\mathcal{P}^{d}$ denotes the algebra of homogeneous polynomials of degree $d$.
} 
ProOF. Let us denote by $n$ the natural representation of $G$ and $n^{\vee}$ its contragredient. By definition,

$$
\psi_{\boldsymbol{\alpha}, I_{\sigma}}\left(g_{1}, \ldots, g_{r}\right)=\operatorname{tr}\left(\bigotimes_{i=1}^{r} n\left(g_{i}\right)^{\otimes p_{i}} \otimes n^{\vee}\left(g_{i}\right)^{\otimes q_{i}} \circ I_{\sigma}\right) .
$$

Each factor $n\left(g_{i}\right)^{\otimes p_{i}} \otimes n^{\vee}\left(g_{i}\right)^{\otimes q_{i}}$ corresponds, through (11) with $p=p_{1}$ and $q=q_{1}$, to $n\left(g_{i}\right)^{\otimes p_{i}} \otimes n\left(g_{i}^{-1}\right)^{\otimes q_{i}}$, by definition of the contragredient. Thus,

$$
\psi_{\boldsymbol{\alpha}, I_{\sigma}}\left(g_{1}, \ldots, g_{r}\right)=\operatorname{tr}\left(\bigotimes_{i=1}^{r} n\left(g_{i}\right)^{\otimes p_{i}} \otimes \bigotimes_{i=1}^{r} n\left(g_{i}^{-1}\right)^{\otimes q_{i}} \circ \pi(\sigma)\right)
$$

where we see now both endomorphisms as elements of $\operatorname{End}\left(V^{\otimes p+q}\right)$. This trace can now easily be evaluated. Before that and for the sake of clarity, let us rename the sequence $\left(g_{1}, \ldots, g_{1}, \ldots, g_{r}, \ldots, g_{r}, g_{1}^{-1}, \ldots, g_{1}^{-1}, \ldots, g_{r}^{-1}, \ldots, g_{r}^{-1}\right)$, where $g_{i}$ appears $p_{i}$ times and $g_{i}^{-1} q_{i}$ times, as $\left(h_{1}, \ldots, h_{p+q}\right)$. Then the tensor product appearing in the last equation is just $h_{1} \otimes \ldots \otimes h_{p+q}$. Hence,

$$
\psi_{\boldsymbol{\alpha}, I_{\sigma}}\left(g_{1}, \ldots, g_{r}\right)=\prod_{\mathcal{C}=\left(a_{1} \ldots a_{k}\right)} \operatorname{tr}\left(h_{a_{1}} \ldots h_{a_{k}}\right),
$$

where the product runs over the cycles of $\sigma$. We claim that each factor in this product is a Wilson loop. To see this, define the functions $j:\{1, \ldots, p+q\} \longrightarrow\{1, r\}$ by

$$
\begin{array}{lll}
j(a)=i & \text { if } \quad p_{1}+\ldots+p_{i-1}<a \leq p_{1}+\ldots+p_{i} \\
& \text { or } \quad p+q_{1}+\ldots+q_{i-1}<a \leq p+q_{1}+\ldots+q_{i}
\end{array}
$$

and $\varepsilon:\{1, \ldots, p+q\} \longrightarrow\{1,-1\}$ such that $\varepsilon(a)$ is +1 if $1 \leq a \leq p$ and -1 if $p+1 \leq a \leq q$. They are designed in such a way that $h_{a}=g_{j(a)}^{\varepsilon(a)}$.

Let us now give a name to the edges of the graph $\mathrm{L}_{\mathrm{r}}$, namely set $E=\left(e_{1}, \ldots, e_{r}\right)$. For each cycle $\mathcal{C}=\left(a_{1} \ldots a_{k}\right)$ of $\sigma$, define a loop $l_{\mathcal{C}}$ in $\mathrm{L}_{\mathrm{r}}$ by $l_{\mathcal{C}}=\left(e_{j\left(a_{k}\right)}^{\varepsilon\left(a_{k}\right)}, \ldots, e_{j\left(a_{1}\right)}^{\varepsilon\left(a_{1}\right)}\right)$. Then the last equality can be rewritten simply as

$$
\psi_{\boldsymbol{\alpha}, I_{\sigma}}\left(g_{1}, \ldots, g_{r}\right)=\prod_{\mathcal{C}=\left(a_{1} \ldots a_{k}\right)} W_{n, l_{\mathcal{C}}}\left(g_{1}, \ldots, g_{r}\right)
$$

and the result is proved.

This proof has a nice graphical representation which allows one to understand very easily the generalization to the orthogonal and symplectic cases.

Let us represent a tensor of $V^{\otimes p} \otimes\left(V^{*}\right)^{\otimes q}$ by a box with $p+q$ oriented legs, $p$ outwards and $q$ inwards. We put inside the box a schematic description of the tensor. For example, the leftmost picture in figure 2 represents a tensor of $V^{*} \otimes V$. It could be labeled by an element of $\operatorname{End}(V)$ or $\operatorname{End}\left(V^{*}\right)$.

The middle picture represents the tensor $\pi((123)) \in \operatorname{End}\left(V^{\otimes 3}\right)$. The rightmost picture represents the same tensor, via the identification ${ }^{6} \operatorname{End}\left(V^{\otimes 3}\right) \simeq \operatorname{End}\left(V^{\otimes 2} \otimes V^{*}\right)$.

\footnotetext{
${ }^{6} \mathrm{We}$ will stay a bit loose about the order of the factors in the tensors. We hope the pictures are clear enough by themselves.
} 

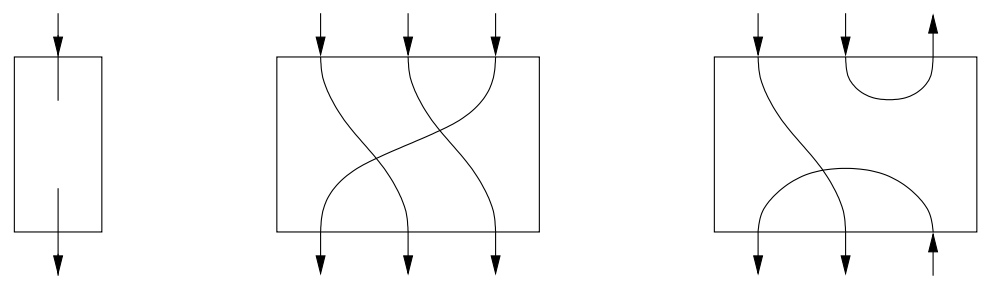

Figure 2: Schematic representation of tensors.

In this representation, tensor product corresponds to juxtaposition of the boxes and a contraction is represented by joining an outcoming leg with an incoming one.

Let us consider a particular case, for example $r=2, p_{1}=q_{2}=0, q_{1}=1$ and $p_{2}=2$. We take the permutation $\sigma=(123)$. Choose $(g, h) \in G^{2}$. The picture corresponding to $\operatorname{tr}\left(n^{\vee}(g) \otimes n(h)^{\otimes 2} \circ I_{\sigma}\right)$ is drawn below (Figure 3 ).

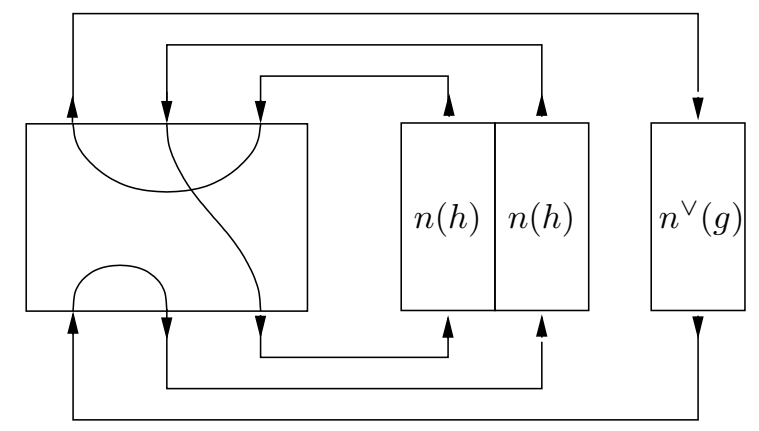

Figure 3: The spin network $\psi_{\left(n^{\vee}, n^{\otimes 2}\right), I_{(123)}}$ on $\mathrm{L}_{2}$ as the Wilson loop $W_{n,\left(e_{1}^{-1}, e_{2}, e_{2}\right)}$.

If one remembers that, through the identification $\operatorname{End}\left(V^{*}\right) \simeq \operatorname{End}(V), n^{\vee}(g)$ corresponds to $n\left(g^{-1}\right)$, it becomes almost evident that the trace we are computing is also a Wilson loop, namely $\operatorname{tr} n\left(g^{-1} h^{2}\right)$.

\section{Orthogonal and symplectic groups}

Let $n \geq 1$ be an integer. Let $G$ be either $O(n), S O(n)$ or $S p(n)$. Recall that, by the two first groups of this list we mean respectively $O_{n} \mathbb{R}$ and $S O_{n} \mathbb{R}$. By the third we mean the subgroup $U(2 n) \cap S p_{2 n} \mathbb{C}$ of $G L_{2 n} \mathbb{C}$, which preserves, via the identification $\mathbb{H}^{n} \simeq \mathbb{C}^{n} \oplus j \mathbb{C}^{n}$, the standard quaternionic Hermitian scalar product on $\mathbb{H}^{n}$. We are going to treat at once the orthogonal and symplectic case, although they are not exactly identical. For example, the space $V$ of the natural representation of $G$ is $\mathbb{C}^{n}$ in the orthogonal case, $\mathbb{C}^{2 n}$ in the symplectic case. We shall use the letter $m$ to denote the dimension of $V$ in both cases. 
In the orthogonal case, we are going to use orthonormal bases of $V$. In the symplectic case, we say that $\left(e_{1}, \ldots, e_{2 n}\right)$ is a standard basis for $V$ if $\left\langle e_{i}, e_{i+n}\right\rangle=1$ for $i=1, \ldots, n$ and $\left\langle e_{i}, e_{j}\right\rangle=0$ if $|i-j| \neq n$.

The situation here differs from the preceding one in two main respects because $G$ preserves a non-degenerate quadratic form $\langle\cdot, \cdot\rangle$ on $V$. First of all, this quadratic form induces an isomorphism $v \mapsto\langle v, \cdot\rangle$ between $V$ and $V^{*}$ which intertwines the natural representation and its contragredient. So, there is no need in this case to consider $V^{*}$. Then, if $\rho$ denotes as before the diagonal action of $G$ on $V^{\otimes d}, \rho(\mathbb{C} G)^{\prime}$ is larger than ${ }^{7}$ $\pi\left(\mathfrak{S}_{d}\right)$. The first fundamental theorem tells us how much larger.

In this section, we will identify freely $\operatorname{End}\left(V^{\otimes d}\right)$ with $V^{\otimes 2 d}$ by saying that $v_{1} \otimes \ldots \otimes v_{2 d}$ transforms $w_{1} \otimes \ldots \otimes w_{d}$ into $\prod_{i=1}^{d}\left\langle v_{i}, w_{i}\right\rangle v_{d+1} \otimes \ldots \otimes v_{2 d}$.

Let $\tau$ be a partition of the set $\{1, \ldots, 2 d\}$ in pairs. Let $\left(e_{1}, \ldots, e_{m}\right)$ be an orthonormal or standard basis of $V$, according to the nature of $G$. We define $J_{\tau} \in \operatorname{End}\left(V^{\otimes d}\right)$ by

$$
J_{\tau}=\sum_{i_{1}, \ldots, i_{2 d}=1}^{m} \prod_{\{k, l\} \in \tau, k<l}\left\langle e_{i_{k}}, e_{i_{l}}\right\rangle e_{i_{1}} \otimes \ldots \otimes e_{i_{2 d}} .
$$

One checks easily that this definition of $J_{\tau}$ does not depend on the choice of the orthonormal basis of $V$ and that $J_{\tau}$ commutes to the action of $G$, that is, $J_{\tau} \in \rho(\mathbb{C} G)^{\prime}=$ $\operatorname{End}_{G}\left(V^{\otimes d}\right)$.

The graphical representation introduced in the preceding section may be helpful to clarify the situation. An example is given by Figure 4. Note that we do not need arrows to distinguish between $V$ and $V^{*}$ anymore, since we are working in tensor powers of $V$ alone.

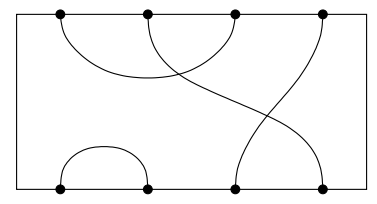

Figure 4: The endomorphism $J_{\tau}$, when $d=4$ and $\tau=\{\{1,3\},\{2,8\},\{4,7\},\{5,6\}\}$.

The following theorem is proved in [3].

Theorem 7.1 (FFT for orthogonal and symplectic groups) The subspace $\operatorname{End}_{G}\left(V^{\otimes d}\right)=\rho(\mathbb{C} G)^{\prime}$ of $\operatorname{End}\left(V^{\otimes d}\right)$ is spanned by the endomorphisms $J_{\tau}$, where $\tau$ runs over the partitions of $\{1, \ldots, 2 d\}$ in pairs.

Remark 7.2 The proof of this theorem is longer than that of Schur-Weyl duality, so we do not give it here. However, it is usually stated and proved for complex Lie groups rather than compact ones. Let us explain how the former can be deduced from the latter.

\footnotetext{
${ }^{7}$ We keep the notation $\pi$ for the action of the symmetric group of any order on the corresponding tensor power of $V$.
} 
If $G$ is $O(n)$ (resp. $S p(n)$ ), let us denote by $G_{\mathbb{C}}$ the group $O_{n} \mathbb{C}$ (resp. $S p_{2 n} \mathbb{C}$ ). Since $G$ is contained in $G_{\mathbb{C}}$, one needs just prove that any $u \in \operatorname{End}_{G}\left(V^{\otimes d}\right)$ is invariant by the whole $G_{\mathbb{C}}$. Via the isomorphism $\operatorname{End}\left(V^{\otimes d}\right) \simeq V^{\otimes 2 d} \simeq\left(V^{*}\right)^{\otimes 2 d}$, we can think of $u$ as a polynomial, that we denote by $\tilde{u}$, in $2 d$ variables on $V$, homogeneous of degree one in each variable, invariant under the action of $G$. This means that, for every $v \in V^{\oplus 2 d}$, the function $\tilde{u}(\cdot v): G_{\mathbb{C}} \longrightarrow \mathbb{C}$ which sends $g$ to $\tilde{u}(g v)$ is constant on $G$. Since, on one hand, this function is polynomial in $g$ and on the other hand, $G$ is Zariski-dense in $G_{\mathbb{C}}$, the function is constant on $G_{\mathbb{C}}$. So, $u$ is invariant by the whole complex orthogonal group.

The theorem for $S O(n)$ follows from that for $O(n)$ just because $\rho(\mathbb{C} S O(n))=\rho(\mathbb{C} O(n))$.

We proceed now as before. Let $p_{1}, \ldots, p_{r}$ be integers. For each $i=1 \ldots r$, let $\alpha_{i}$ denote $V^{\otimes p_{i}}$ and set $\boldsymbol{\alpha}=\left(\alpha_{1}, \ldots, \alpha_{r}\right)$. Set $p=p_{1}+\ldots+p_{r}$. Let $\tau$ be a partition of $\{1, \ldots, 2 r\}$ in pairs.

Proposition 7.3 The spin network $\psi_{\boldsymbol{\alpha}, J_{\tau}}$ on $\mathrm{L}_{\mathrm{r}}$ is a product of Wilson loops.

The proof is very similar to that of Proposition 6.2. We are going to show that, up to some isomorphism, $J_{\tau}$ acts as a permutation operator. For this, define for each $i=1 \ldots p$ $T_{i}=\pi((i, p+i)) \in \operatorname{End}\left(V^{\otimes 2 p}\right) \simeq \operatorname{End}\left(\operatorname{End}\left(V^{\otimes p}\right)\right)$.

Lemma 7.4 Let $\tau$ be a partition of $\{1, \ldots, 2 p\}$ in pairs. There exist $i_{1}, \ldots, i_{k} \in\{1, \ldots, p\}$ and $\sigma \in \mathfrak{S}_{p}$ such that $T_{i_{1}} \circ \ldots \circ T_{i_{k}}\left(J_{\tau}\right)=\pi(\sigma)$.

Remark 7.5 It is worth saying what this lemma means graphically, because this is much simpler than the aspect of the proof might suggest. Let us represent, as we did in Figure 4. a partition like $\tau$ as a pairing of $2 p$ points by $p$ lines. We put the points $1, \ldots, p$ on the top edge of a box and $p+1, \ldots, 2 p$ on the bottom edge, with $p+i$ below $i$. Then the lemma says that, by switching the positions of $i$ and $p+i$ for some well-chosen $i$ 's without changing the pairing $\tau$, we can make sure that every line connects a point on the top edge with a point on the bottom edge. The diagram one gets in that way corresponds to a permutation operator.

Proof. It is convenient in this proof to think of $\tau$ as a fixed-point free involution of $\{1, \ldots, 2 p\}$. Let $\theta_{1}, \ldots, \theta_{p}$ denote the transpositions $(1, p+1), \ldots,(p, 2 p)$. Then, given some integers $i_{1}, \ldots, i_{k}$ between 1 and $p$, one checks easily that

$$
T_{i_{1}} \circ \ldots \circ T_{i_{k}}\left(J_{\tau}\right)=J_{\theta_{i_{1}} \ldots \theta_{i_{k}} \tau \theta_{i_{1}} \ldots \theta_{i_{k}}}
$$

where the product in the subscript of $J$ is a composition of permutations of $\{1, \ldots, 2 p\}$. On the other hand, if $\sigma$ is a permutation of $\{1, \ldots, p\}$ and $\tau$ pairs $i$ with $\sigma(i)+p$ for each $i=1 \ldots p$, then $J_{\tau}=\pi(\sigma)$. Thus, the lemma will be proved if we show that, for some $i_{1}, \ldots, i_{k}$ between 1 and $p$ and some $\sigma \in \mathfrak{S}_{p}, \theta_{i_{1}} \ldots \theta_{i_{k}} \tau \theta_{i_{1}} \ldots \theta_{i_{k}}$ pairs $i$ with $\sigma(i)+p$ for $i=1 \ldots p$. 
To do this, set $\theta=\theta_{1} \ldots \theta_{p}$. The map $\theta \tau$ acts on $\{1, \ldots, 2 p\}$ and we are interested in its orbits. In particular, observe that $\{1, \ldots, p\}$ is a reunion of orbits of $\theta \tau$ if and only if there exists $\sigma \in \mathfrak{S}_{p}$ such that $\tau$ pairs $i$ with $\sigma(i)+p$ for all $i=1 \ldots p$.

We define by induction a sequence of cycles, that is, of cyclic permutations, on $\{1, \ldots, p\}$ as follows.

Set $x_{1}=1$ and let $\mathcal{O}_{1}$ be the orbit of $x_{1}$ under $\theta \tau$, endowed with its cyclic order. Let $m_{p}:\{1, \ldots, 2 p\} \longrightarrow\{1, \ldots, p\}$ be the map which sends $i$ and $i+p$ to $i, i=1 \ldots p$. Define the cycle $\mathcal{C}_{1}=m_{p}\left(\mathcal{O}_{1}\right)$ and set $x_{2}=\min \left(\{1, \ldots, p\}-\mathcal{C}_{1}\right)$. Then, given $\mathcal{C}_{1}, \ldots, \mathcal{C}_{n-1}$ and $x_{n}$, define $\mathcal{O}_{n}$ as the orbit of $x_{n}, \mathcal{C}_{n}=m_{p}\left(\mathcal{O}_{n}\right)$ and $x_{n+1}=\min \left(\{1, \ldots, p\}-\left(\mathcal{C}_{1} \cup \ldots \cup \mathcal{C}_{n}\right)\right)$. This procedure stops when the cycles $c_{1}, \ldots, \mathcal{C}_{n}$ cover $\{1, \ldots, p\}$. These cycles are disjoint by construction and we see each of them as an element of $\mathfrak{S}_{p}$. Let us call $\sigma$ their product.

Now for each $i=1 \ldots p$, one and only one of the two elements $i$ and $i+p$ belong to $\mathcal{O}_{1} \cup \ldots \cup \mathcal{O}_{n}$. Set $\varepsilon_{i}=0$ if it is $i, \varepsilon_{i}=1$ if it is $i+p$. Define $\tilde{\tau}=\left(\prod_{i=1}^{p} \theta_{i}^{\varepsilon_{i}}\right) \tau\left(\prod_{i=1}^{p} \theta_{i}^{\varepsilon_{i}}\right)$. It is easily checked that the iterates of $\theta \tilde{\tau}$ preserve $\{1, \ldots, p\}$ and in fact that $\tilde{\tau}$ pairs $i$ with $\sigma(i)+p$ for $i=1 \ldots p$. The lemma is proved, by taking for $i_{1}, \ldots, i_{k}$ those integers $i$ such that $\varepsilon_{i}=1$.

The elements of $G$ have a simple behaviour under the transposition operators $T_{i}$.

Lemma 7.6 Consider the following isomorphism:

$$
T: \operatorname{End}(V) \simeq V^{*} \otimes V \stackrel{\langle,\rangle \otimes \operatorname{Id}}{\longrightarrow} V \otimes V \stackrel{\pi((12))}{\longrightarrow} V \otimes V \stackrel{\langle,\rangle \otimes \operatorname{Id}}{\longrightarrow} V^{*} \otimes V \simeq \operatorname{End}(V)
$$

Let $g$ be an element of $G$. Then $T(g)=\varepsilon g^{-1}$, where $\varepsilon=1$ if $G$ is orthogonal and $\varepsilon=-1$ if $G$ is symplectic.

Proof. If $v$ belongs to $V$ and $\phi$ to $V^{*}$, let us denote by $\tilde{v}$ and $\tilde{\phi}$ the corresponding elements of $V^{*}$ and $V$ respectively, so that $\tilde{v}=\langle v, \cdot\rangle$ and $\phi=\langle\tilde{\phi}, \cdot\rangle$.

Let $g=\sum_{i} \phi_{i} \otimes v_{i}$ be an element of $G \subset \operatorname{End}(V)$. Then $T(g)=\sum_{i} \tilde{v}_{i} \otimes \tilde{\phi}_{i}$. Now let $u$ and $w$ be two elements of $V$. One has

$$
\langle g u, w\rangle=\left\langle\sum_{i} \phi_{i}(u) v_{i}, w\right\rangle=\sum_{i} \tilde{v}_{i}(w)\left\langle\tilde{\phi}_{i}, u\right\rangle=\varepsilon\left\langle u, \sum_{i} \tilde{v}_{i}(w) \tilde{\phi}\right\rangle=\langle u, \varepsilon T(g)(w)\rangle,
$$

where $\varepsilon$ equals plus or minus one, according to the symmetry of the form $\langle\cdot, \cdot\rangle$. Since this form is non-degenerate and preserved by $g$, the result follows.

Proof of Proposition 7.3. Let $g_{1}, \ldots, g_{r}$ be $r$ elements of $G$. By definition,

$$
\psi_{\boldsymbol{\alpha}, J_{\tau}}\left(g_{1}, \ldots, g_{r}\right)=\operatorname{tr}\left(g_{1}^{\otimes p_{1}} \otimes \ldots \otimes g_{r}^{\otimes p_{r}} \circ J_{\tau}\right) \text {. }
$$

By Lemma [7.4, there exist $i_{1}, \ldots, i_{k} \in\{1, \ldots, p\}$ and $\sigma \in \mathfrak{S}_{p}$ such that $T_{i_{1}} \circ \ldots \circ T_{i_{k}}\left(J_{\tau}\right)=$ $\pi(\sigma)$, or equivalently, $J_{\tau}=T_{i_{1}} \circ \ldots \circ T_{i_{k}}(\pi(\sigma))$, since $T_{i}^{2}=1$. 
Now, observe that, for $u$ and $u^{\prime}$ in $\operatorname{End}\left(V^{\otimes p}\right)$, one has $\operatorname{tr}\left(u \circ T_{i}\left(u^{\prime}\right)\right)=\operatorname{tr}\left(T_{i}(u) \circ u^{\prime}\right)$ for all $i=1 \ldots p$. Hence, we have

$$
\psi_{\boldsymbol{\alpha}, J_{\tau}}\left(g_{1}, \ldots, g_{r}\right)=\operatorname{tr}\left(T_{i_{1}} \circ \ldots \circ T_{i_{k}}\left(g_{1}^{\otimes p_{1}} \otimes \ldots \otimes g_{r}^{\otimes p_{r}}\right) \circ \pi(\sigma)\right) .
$$

For the sake of clarity, let us rename the sequence $\left(g_{1}, \ldots, g_{1}, \ldots, g_{r}, \ldots, g_{r}\right)$, where $g_{i}$ appears $p_{i}$ times, as just $\left(h_{1}, \ldots, h_{p}\right)$. Thus, $g_{1}^{\otimes p_{1}} \otimes \ldots \otimes g_{r}^{\otimes p_{r}}$ equals $h_{1} \otimes \ldots \otimes h_{p}$. Now, by Lemma 7.6. we have

$$
T_{i_{1}} \circ \ldots \circ T_{i_{k}}\left(h_{1} \otimes \ldots \otimes h_{p}\right)=\varepsilon^{k} h_{1}^{\varepsilon_{1}} \otimes \ldots \otimes h_{p}^{\varepsilon_{p}},
$$

where now $\varepsilon_{i}=-1$ if $i$ appears in the list $i_{1}, \ldots, i_{k}$ and $\varepsilon_{i}=1$ otherwise $^{8}$. Here again, $\varepsilon=1$ in the orthogonal case, -1 in the symplectic one.

Now we finish the proof just as that of Proposition 6.2. Indeed,

$$
\begin{aligned}
\psi_{\boldsymbol{\alpha}, J_{\tau}}\left(g_{1}, \ldots, g_{r}\right) & =\varepsilon^{k} \operatorname{tr}\left(h_{1}^{\varepsilon_{1}} \otimes \ldots \otimes h_{p}^{\varepsilon_{p}} \circ \pi(\sigma)\right) \\
& =\varepsilon^{k} \prod_{\mathcal{C}=\left(a_{1} \ldots a_{k}\right)} \operatorname{tr}\left(h_{a_{1}}^{\varepsilon_{a_{1}}} \ldots h_{a_{k}}^{\varepsilon_{a_{k}}}\right),
\end{aligned}
$$

where the product runs over the decomposition of $\sigma$ in cycles. Each factor in this product is a Wilson loop. Indeed, let us define $j:\{1, \ldots, p\} \longrightarrow\{1, \ldots r\}$ by

$$
j(a)=\min \left\{i: a \leq p_{1}+\ldots+p_{i}\right\} .
$$

Then by definition, $h_{a}=g_{j(a)}$. If $E=\left\{e_{1}, \ldots, e_{r}\right\}$ denotes the set of edges of the graph $\mathrm{L}_{\mathrm{r}}$, then we can define for every cycle $\mathcal{C}=\left(a_{1} \ldots a_{k}\right)$ of $\sigma$ the loop $l_{\mathcal{C}}=\left(e_{j\left(a_{1}\right)}^{\varepsilon_{a_{1}}}, \ldots, e_{j\left(a_{k}\right)}^{\varepsilon_{a_{k}}}\right)$. With this notation, we have proved that

$$
\psi_{\boldsymbol{\alpha}, J_{\tau}}\left(g_{1}, \ldots, g_{r}\right)=\varepsilon^{k} \prod_{\mathcal{C}=\left(a_{1} \ldots a_{k}\right)} W_{n, l_{\mathcal{C}}}
$$

where $n$ denotes the natural representation. This proves the proposition.

\section{References}

[1] John C. Baez. Spin networks in gauge theory. Adv. Math., 117(2):253-272, 1996.

[2] Theodor Bröcker and Tammo tom Dieck. Representations of compact Lie groups. Springer-Verlag, New York, 1995. Translated from the German manuscript, Corrected reprint of the 1985 translation.

[3] William Fulton and Joe Harris. Representation theory, volume 129 of Graduate Texts in Mathematics. Springer-Verlag, New York, 1991. A first course, Readings in Mathematics.

\footnotetext{
${ }^{8}$ This new $\varepsilon_{i}$ is minus twice the one defined in the proof of Lemma 7.4 plus one.
} 
[4] Serge Lang. Algebra, volume 211 of Graduate Texts in Mathematics. Springer-Verlag, New York, third edition, 2002.

[5] Alain Robert. Introduction to the representation theory of compact and locally compact groups, volume 80 of London Mathematical Society Lecture Note Series. Cambridge University Press, Cambridge, 1983.

[6] Ambar Sengupta. The Yang-Mills measure for $S^{2}$. J. Funct. Anal., 108(2):231-273, 1992.

[7] Ambar Sengupta. Gauge invariant functions of connections. Proc. Amer. Math. Soc., 121(3):897-905, 1994.

[8] Lee Smolin. The physics of spin networks. In The geometric universe (Oxford, 1996), pages 291-304. Oxford Univ. Press, Oxford, 1998. 